\title{
MULTI-LOOK PROCESSING OF HIGH-RESOLUTION SAS DATA FOR IMPROVED TARGET DETECTION PERFORMANCE
}

\author{
David P. Williams \\ NATO STO Centre for Maritime \\ Research and Experimentation (CMRE) \\ La Spezia, Italy \\ david.williams@cmre.nato.int
}

\author{
Alan J. Hunter* \\ University of Bath \\ Department of Mechanical Engineering \\ Bath, United Kingdom \\ a.j.hunter@bath.ac.uk
}

\begin{abstract}
The rich content of synthetic aperture sonar (SAS) data is typically used to generate imagery with resolution as high as theoretically possible. But when the ultimate purpose of the imagery is for detecting objects with sizes large compared to the resolution cell, exploiting the raw data in alternative ways can be more useful. We first show how multiple lower-resolution SAS images (i.e., "looks") can be obtained in an efficient, principled manner by band-limiting the image wavenumber spectrum of a full-resolution SAS image. By combining these multiple looks (from different aspect and frequency bands), a despeckled image that enjoys greater (target) signal to (seabed) reverberation ratio can be produced. On a large set of real SAS data collected at sea, it is demonstrated how better target detection performance of underwater mines can be achieved by using the lower-resolution despeckled imagery rather than single-look, maximum-resolution imagery.
\end{abstract}

Index Terms - Target detection, synthetic aperture sonar, despeckling, multi-look processing, high-resolution imagery

\section{INTRODUCTION}

Synthetic aperture sonar (SAS) works by coherently summing received acoustic signals of overlapping elements in an array, and it provides an order-of-magnitude improvement in resolution over simple (real aperture) side-scan sonar data [1]. The resulting high-resolution SAS imagery provides a detailed view of the seafloor that makes detection of proud targets (e.g., mines) possible.

The rich content of SAS data is typically used to generate imagery with resolution as high as theoretically possible. However, when the sizes of the targets to be detected are very large compared to the resolution cell of the image, there can be an excess of bandwidth available. Under these circumstances when there are many pixels on target, it can make more sense to sacrifice resolution in order to generate multiple (lower-resolution) images, or "looks," of the scene. This

\footnotetext{
*Work performed while at CMRE.
}

multi-look processing, in which reduced aspect bands or frequency bands are used to create each "subimage," provides a promising alternative for exploiting the rich content of the raw sonar data.

In this work, we show how this multi-look processing can be used to enable a form of despeckling that improves target detection performance beyond that which can be achieved with a single maximum-resolution image. That is, by combining multiple looks of a scene, a despeckled image that enjoys greater (target) signal to (seabed) reverberation ratio can be produced. Importantly, we show how this approach can be performed efficiently with SAS data such that computationally intensive processing steps are avoided.

Multi-look processing has been used in the more mature synthetic aperture radar (SAR) community to perform despeckling [2], but the approach has not been seriously pursued in the underwater domain with sonar imagery. Previous work on despeckling in sonar images has instead focused exclusively on applying various filters to the (fullresolution) imagery, including basic averaging filters (i.e., mean, median) $[3,4]$, classical adaptive filters (e.g., Lee, Kuan, Frost) [4-6], wavelet-based filters [4-7], and stochastic matched filters [8]. To our knowledge, this work is the first to employ explicit multi-look processing with the intent of despeckling sonar imagery. The related topic of sub-aperture imaging (i.e., multiple looks via banding in aspect only) with sonar has previously been explored only in the context of $360^{\circ}$ circular SAS for object identification [9].

\section{MULTI-LOOK SAS DESPECKLING}

It is possible to exploit the high-resolution information contained in the raw sonar data in different ways. Rather than forming a single maximum-resolution SAS image, one can construct multiple lower-resolution subimages (i.e., "looks") from the same raw data. This is more feasible now because of the significant bandwidths and complex signals associated with synthetic apertures vis-à-vis side-scan sonars. Provided one already possesses the full-resolution (complex) SAS im- 
age, this alternative formulation can be obtained via the following computationally efficient procedure.

The full image resolution in the along-track and range dimensions are related, respectively, to the spatial and temporal bandwidths of the sonar by $\delta_{x}=D / 2$ and $\delta_{y}=c / 2 B$, where $D$ is the along-track dimension of the largest (transducer) aperture, $B$ is the signal bandwidth in $\mathrm{Hz}$, and $c \approx 1500 \mathrm{~m} / \mathrm{s}$ is the propagation speed.

We effectively degrade the resolution by factors of $\alpha_{x}$ and $\alpha_{y}$ in each of the respective image dimensions by bandlimiting the image wavenumber spectrum, i.e.,

$$
\begin{aligned}
i^{(m n)}(x, y)= & \int_{-\infty}^{\infty} I^{(m n)}\left(k_{x}, k_{y}\right) \\
& \times \exp \left(j\left(k_{x} x+k_{y} y\right)\right) \mathrm{d} k_{x} \mathrm{~d} k_{y}
\end{aligned}
$$

where

$$
\begin{aligned}
I^{(m n)}\left(k_{x}, k_{y}\right)= & \operatorname{win}\left(\frac{\delta_{x}^{\prime}}{2 \pi} k_{x}-k_{x}^{(m n)}\right) \\
& \times \operatorname{win}\left(\frac{\delta_{y}^{\prime}}{2 \pi} k_{y}-k_{y}^{(m n)}\right) I\left(k_{x}, k_{y}\right)
\end{aligned}
$$

is the band-limited spectrum with wavenumbers $k_{x}$ and $k_{y}$, $\delta_{x}^{\prime}=\alpha_{x} \delta_{x}$ and $\delta_{y}^{\prime}=\alpha_{y} \delta_{y}$ are the dimensions of the degraded resolution cell, win $(z)$ is a window function that is non-zero only on the interval $z \in[-0.5,0.5], k_{x}^{(m n)}$ and $k_{y}^{(m n)}$ are scalar offsets to specify the centers of the $m n$th band, and

$$
I\left(k_{x}, k_{y}\right)=\int_{-\infty}^{\infty} i(x, y) \exp \left(-j\left(k_{x} x+k_{y} y\right)\right) \mathrm{d} k_{x} \mathrm{~d} k_{y}
$$

is the spectrum of the full-resolution image $i(x, y)$. We then decimate the image pixels accordingly by the same factors $\alpha_{x}$ and $\alpha_{y}$.

By selecting different bands of the spectrum (via $k_{x}^{(m n)}$ and $k_{y}^{(m n)}$ ), multiple lower-resolution subimages can be generated. When the bands collectively span the full spectrum, all the information content in the raw data is still being exploited, only in a different manner than usual. A single final despeckled image is produced from $M N$ subimages by summing incoherently, i.e.,

$$
i^{\prime}(x, y)=\sum_{m=1}^{M} \sum_{n=1}^{N}\left|i^{(m n)}(x, y)\right| .
$$

Importantly, the computationally intensive processing required to generate the initial full-resolution SAS image [10] need not be performed to produce the lower-resolution subimages.

The above process is illustrated visually in the following. One example full-resolution SAS image of a target, along with its image wavenumber spectrum, is shown in Fig. 1. (The target was extracted from a larger image scene covering an area of $50 \mathrm{~m} \times 110 \mathrm{~m}$.) For this image, the result of dividing the full spectrum into non-overlapping bands when $\alpha_{x}=4$ and $\alpha_{y}=4$ is shown in Fig. 2; the corresponding lowerresolution SAS subimages are then shown in Fig. 3. Example despeckled images obtained from different factors $\alpha_{x}$ and $\alpha_{y}$ are shown in Fig. 4.

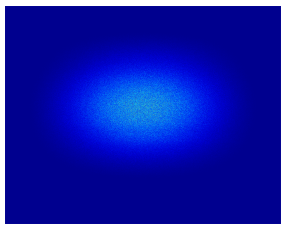

(a)

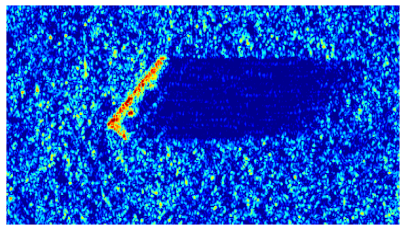

(b)
Fig. 1. For the full-resolution case, (a) the image wavenumber spectrum where the $x$-axis corresponds to wavenumber $k_{x}$ and the $y$-axis corresponds to wavenumber $k_{y}$, and (b) the corresponding SAS image, where the $y$-axis corresponds to along-track direction and the $x$-axis corresponds to range. The SAS image covers an area of $5 \mathrm{~m} \times 9 \mathrm{~m}$.

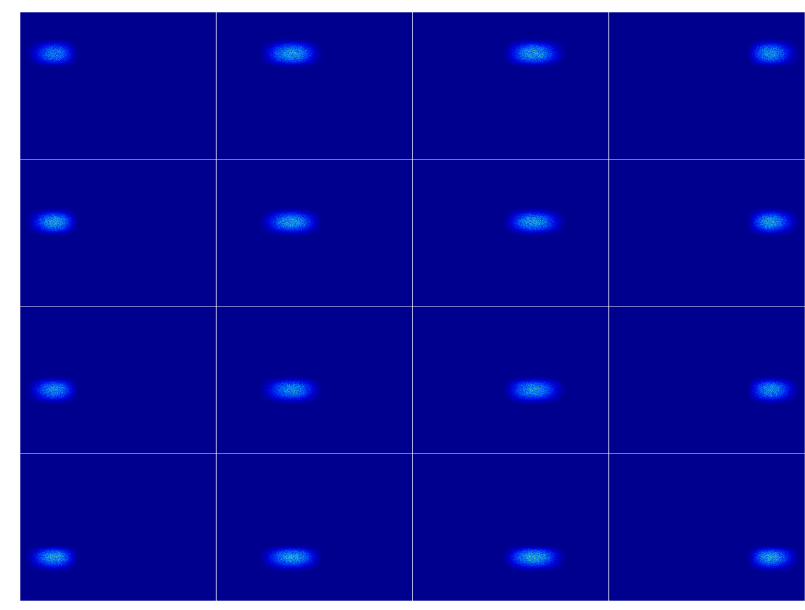

Fig. 2. For a given SAS image, the image wavenumber spectra where the $x$-axis corresponds to wavenumber $k_{x}$ and the $y$-axis corresponds to wavenumber $k_{y}$, when the subimages were band-limited in both $k_{y}$ and $k_{x}$, such that the range and along-track resolutions were each degraded by a factor of 4 . Each column shows the images obtained from using a different aspect band. Each row shows the images obtained from using a different frequency band.

\section{EXPERIMENTAL RESULTS}

In this work, the above procedure is exploited to improve target-detection performance beyond that which can be achieved using a single maximum-resolution image. The data used in the study were collected by the CMRE's SASequipped autonomous underwater vehicle (AUV) called MUSCLE. This SAS has an aperture length of $D=5 \mathrm{~cm}$ 


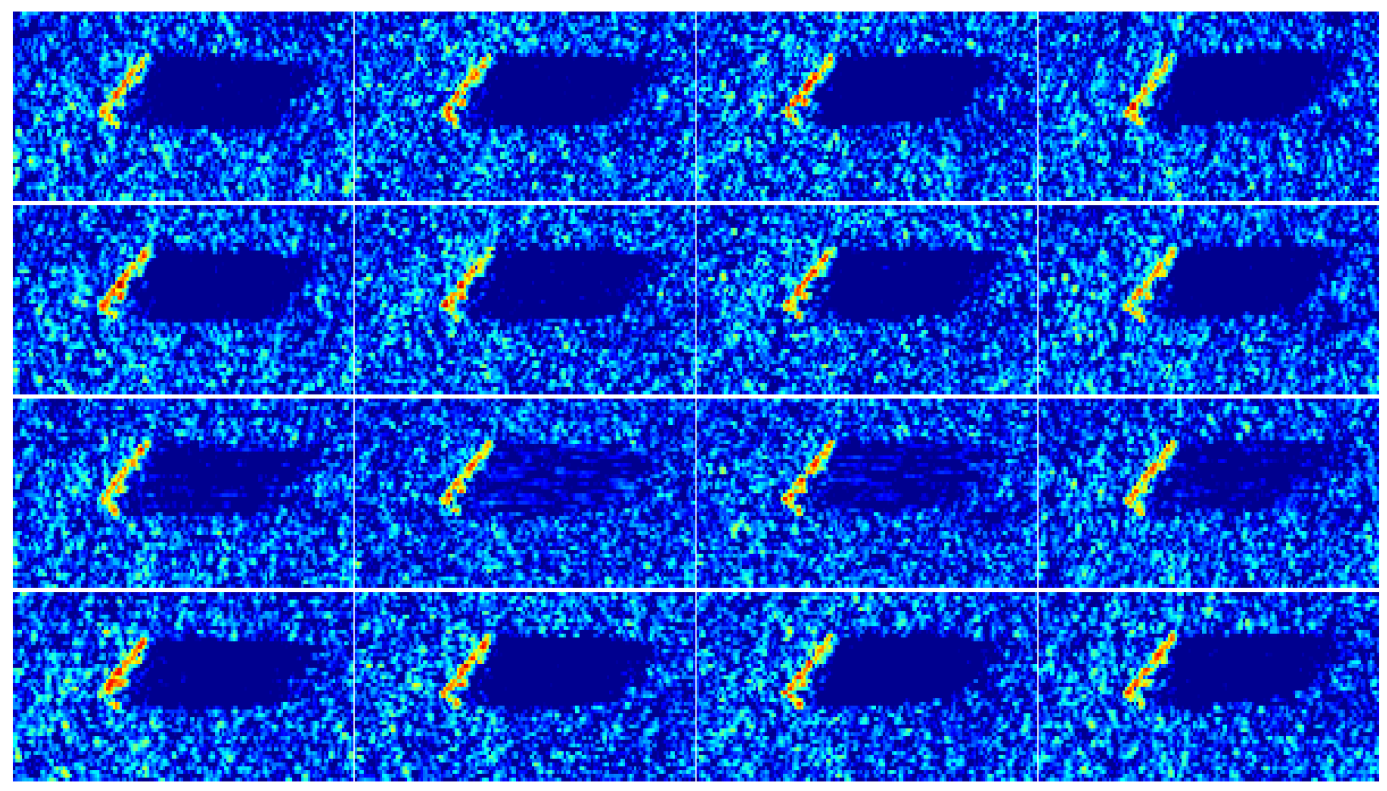

Fig. 3. SAS subimages, where the $y$-axis corresponds to along-track direction and the $x$-axis corresponds to range, obtained from using different aspect and frequency bands. Each image covers an area of $5 \mathrm{~m} \times 9 \mathrm{~m}$, and the resolution has been degraded by a factor of 4 in each direction from the full-resolution case. Each column shows the images obtained from using a different aspect band. Each row shows the images obtained from using a different frequency band.

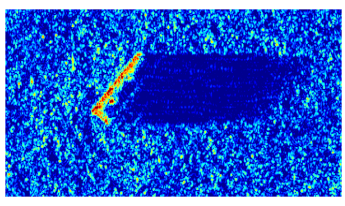

(a)

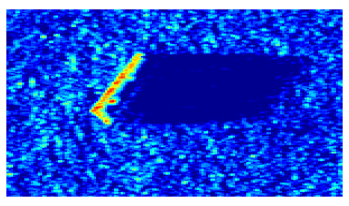

(b)

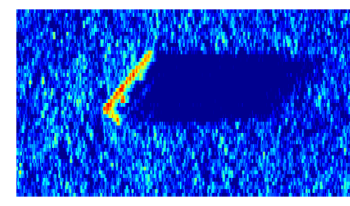

(c)

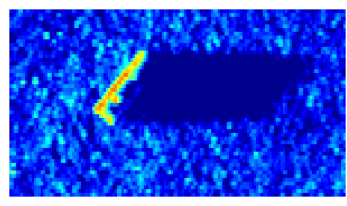

(d)

Fig. 4. Despeckled images obtained from averaging lower-resolution subimages, where the $y$-axis corresponds to along-track direction and the $x$-axis corresponds to range. Each image covers an area of $5 \mathrm{~m} \times 9 \mathrm{~m}$. (a) The original full-resolution image $\left(\alpha_{x}=1\right.$ and $\alpha_{y}=1$ ); (b) when the (four) contributing images were band-limited in $k_{y}$, such that the range resolution was degraded by a factor of $\alpha_{y}=4$; (c) when the (four) contributing images were band-limited in $k_{x}$, such that the along-track resolution was degraded by a factor of $\alpha_{x}=4$; and (d) when the (sixteen) contributing images (shown in Fig. 3) were bandlimited in both $k_{y}$ and $k_{x}$, such that the range and along-track resolutions were each degraded by a factor of 4 .

and bandwidth of $B=60 \mathrm{kHz}$, which yields a (full) resolution cell of $\delta_{x}=2.5 \mathrm{~cm}$ in the along-track dimension and $\delta_{y}=1.25 \mathrm{~cm}$ in the range dimension.

The data were collected during five major experiments conducted at sea by NURC/CMRE between 2009 and 2012 at different geographical sites. The data comprises over 1100 images collectively spanning over $6 \mathrm{~km}^{2}$ of seabed and containing over 1500 mine-like targets (i.e., detection opportunities).

Here, each full-resolution image in the data set is degraded to a series of different along-track resolutions and range resolutions by band-limiting the spectrum. We consider the cases when the image-resolution degradation factors $\alpha_{x}$ and $\alpha_{y}$ are each taken from the set $A=\{1,2,4,6,8,10\}$. For a given $\alpha_{x}$ and $\alpha_{y}$ pair, $\alpha_{x} \alpha_{y}$ unique low-resolution looks are produced by dividing the full spectrum into $\alpha_{x}$ (non-overlapping) aspect bands and $\alpha_{y}$ (non-overlapping) frequency bands. These multiple looks are then averaged to form a single despeckled image. (For example, when $\alpha_{x}=6$ and $\alpha_{y}=10$, the despeckled image is the average of 60 lower-resolution looks.)

A cascaded target-detection algorithm [11] that exploits integral images [12] is then applied to every despeckled image. This algorithm first searches for strong shadow regions (where the sonar returns are lower than the local surrounding seabed) consistent with targets, and then searches for strong highlight regions (where the sonar returns are higher than the local surrounding seabed). For each image-resolution case, the numbers of targets correctly detected and false alarms generated are computed. A summary of these detection per- 


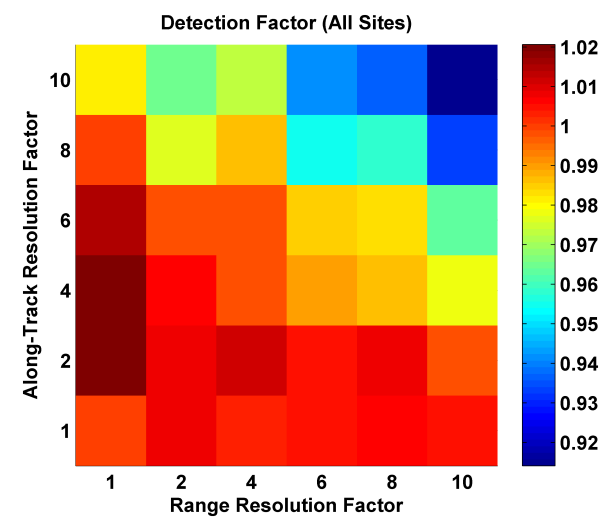

(a)

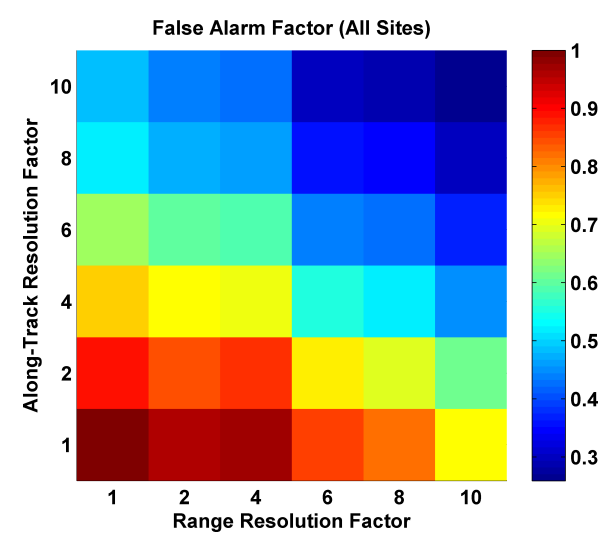

(b)

Fig. 5. Detection performance, across all sites, as a function of along-track and range resolution factors of the subimages used for despeckling. (a) The detection factor expresses the probability of detection divided by that of the full-resolution case. (b) The false alarm factor expresses the number of false alarms divided by that of the full-resolution case (e.g., a false alarm factor of 0.5 indicates the case had half as many false alarms as the full-resolution case).

formance results across all sites is shown in Fig. 5.

It should be noted that the case in which $\alpha_{x}=1$ and $\alpha_{y}=1$ corresponds to the full-resolution image (i.e., with no despeckling). Detection performance is reported as a function of this case to more clearly show the effects of the proposed despeckling procedure. As can be seen in Fig. 5, as the resolution factors increase, the false alarm rate decreases (which is desired) but the target detection probability also sometimes decreases (which is not desired). But it is the relative rate at which these quantities change that is of particular interest.

To determine whether the proposed despeckling procedure achieves better performance than simply using the maximum-resolution imagery, one can examine the result of raising the detection threshold (i.e., effectively moving along the receiver operating characteristic (ROC) curve) with the latter approach since this also decreases both the false alarm

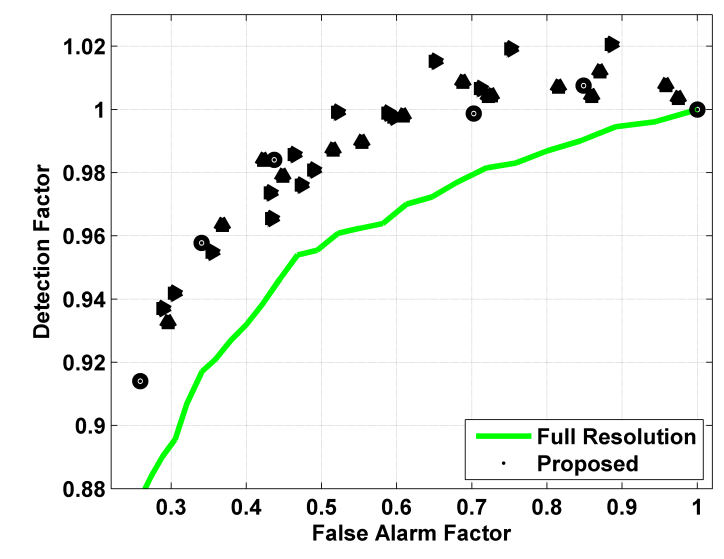

Fig. 6. Detection performance, across all sites, of the proposed despeckling for different along-track and range resolution factors, compared to varying the detection threshold in the full-resolution case. Each black marker corresponds to one case from Fig. 5.

rate and the detection probability. As can be seen in Fig. 6, the proposed despeckling approach is able to maintain a higher probability of detection than the maximum-resolution approach for a given false alarm rate. In particular, for the data considered, the despeckling is able to decrease the false alarm rate by $60 \%$ while maintaining the same probability of detection. Moreover, when the resolution factors are relatively small, the despeckling approach actually raises the probability of detection above that which was achievable using the maximum-resolution imagery. This result can be attributed to the improved (target) signal to (seabed) reverberation ratio that the despeckling engenders.

These results suggest that for detecting mine-like targets in SAS imagery - where the sizes of the objects are much greater than the size of the maximum-resolution cell - the high-resolution content of the data is better exploited by the proposed multi-look despeckling approach.

\section{CONCLUSION}

A computationally efficient procedure to generate multiple lower-resolution looks - and subsequently a despeckled image - from high-resolution sonar data was proposed. It was then demonstrated on a large set of real SAS data that this alternative use of high-resolution information could enable improved detection performance for underwater mines. Future work will examine the performance of this approach as a function of target type and environmental conditions.

The promising results obtained here hopefully will inspire other researchers to exploit the high-resolution content of sonar data in alternative ways for other image-processingbased tasks. 


\section{REFERENCES}

[1] M. Hayes and P. Gough, "Broad-band synthetic aperture sonar," IEEE Journal of Oceanic Engineering, vol. 17, no. 1, pp. 80-94, 1992.

[2] C. Jakowatz, D. Wahl, P. Eichel, D. Ghiglia, and P. Thompson, Spotlight-Mode Synthetic Aperture Radar: A Signal Processing Approach, vol. 101, Kluwer Academic Publishers Norwell, MA, 1996.

[3] K. Jespersen, H. Sorensen, B. Zerr, B. Stage, and B. Haugsted, "Sonar image enhancements for improved detection of sea mines," in AeroSense' 99. International Society for Optics and Photonics, 1999, pp. 552-558.

[4] O. Lopera, R. Heremans, A. Pizurica, and Y. Dupont, "Filtering speckle noise in SAS images to improve detection and identification of seafloor targets," in IEEE International Waterside Security Conference (WSS), 2010, pp. 1-4.

[5] A. Isar, D. Isar, S. Moga, J. Augustin, and X. Lurton, "Multi-scale MAP despeckling of sonar images," in IEEE OCEANS Europe, 2005, vol. 2, pp. 1292-1297.

[6] S. Karabchevsky, D. Kahana, O. Ben-Harush, and H. Guterman, "FPGA-based adaptive speckle suppression filter for underwater imaging sonar," IEEE Journal of Oceanic Engineering, vol. 36, no. 4, pp. 646-657, 2011.
[7] E. Sang, Z. Shen, H. Bian, and Y. Li, “Sonar image denoising based on HMT model in morphological wavelet domain," in IEEE International Conference on Image Analysis and Signal Processing (IASP), 2010, pp. 214218.

[8] P. Courmontagne, "A review on stochastic matched filter based denoising methods for SAS images despeckling," in IEEE OCEANS Europe, 2007, pp. 1-6.

[9] T. Marston, J. Kennedy, and P. Marston, "Coherent and semi-coherent processing of limited-aperture circular synthetic aperture (CSAS) data," in IEEE OCEANS, 2011, pp. 1-6.

[10] A. Bellettini and M. Pinto, "Theoretical accuracy of synthetic aperture sonar micronavigation using a displaced phase-center antenna," IEEE Journal of Oceanic Engineering, vol. 27, no. 4, pp. 780-789, 2002.

[11] D. Williams, "Fast target detection in synthetic aperture sonar imagery: A new algorithm and large-scale performance analysis," IEEE Journal of Oceanic Engineering, vol. 40, no. 1, pp. 71-92, 2015.

[12] P. Viola and M. Jones, "Robust real-time object detection," International Journal of Computer Vision, vol. 57, no. 2, pp. 137-154, 2004. 